\title{
ELASTO-PLASTIC STABILITY OF THIN PLATES SUBJECTED TO COMPLEX LOADING PROCESS
}

\author{
DAO HUY BICH \\ Hanoi National University
}

SUMMARY. Analysing an elastio-plastic stability problem, the complex loading process acted on the body has an essential influence on the values of critical loads. For clearing up this effect, in the present paper the general elasto - plastic process theory, theory of process with average curvature and the simple loading process theory are applied in to the consideration of the mentioned problem. A numerical comparison is given.

\section{§1. RELATIONS DEFINING THE VALUES OF CRITICAL LOADS}

In [2] a new approach to the stability problem of plates subjected to arbitrary complex loading was given. Now consider the more general case, when there exists a pre-buckling plane stress state in the plate as follows.

$$
\begin{aligned}
\sigma_{11} & =-p, \quad \sigma_{22}=-q, \quad \sigma_{12}=-\tau, \quad \sigma_{33}=\sigma_{23}=\sigma_{13}=0 \\
\sigma_{u} & =\left(p^{2}-p q+q^{2}+3 \tau^{2}\right)^{1 / 2} .
\end{aligned}
$$

Respectively, the arc - length of the deformation trajectory is evaluated from

$$
\frac{d s}{d t}=\frac{2}{\sqrt{3}}\left(\dot{\varepsilon}_{11}^{2}+\dot{\varepsilon}_{22}^{2}+\dot{\varepsilon}_{11} \dot{\varepsilon}_{12}+\dot{\varepsilon}_{12}^{2}\right)^{1 / 2}=F(s, p, q, \tau)
$$

where $\dot{\varepsilon}_{i j}$ are defined through $p, q, \tau$ according to the elasto - plastic process theory.

The post - buckling deformation processes may be arbitrary complicated, so that the possible stress - strain increments are defined by the same theory

$$
\delta \sigma_{i j}=\frac{2}{3} A\left(\delta \varepsilon_{i j}+\delta_{i j} \delta \varepsilon_{k k}\right)+(P-A) \frac{\sigma_{k \ell} \delta \varepsilon_{k \ell}}{\sigma_{u}^{2}} \sigma_{i j}
$$

where

$$
\begin{aligned}
A & =-\frac{\sigma_{u} f}{\sin \theta}=\frac{\sigma_{u}}{s}\left[1+\left(\frac{3 G s}{\sigma_{u}}-1\right) \frac{(1-\cos \theta)}{2}\right]= \\
& =\frac{1}{2}\left(3 G+\frac{\sigma_{u}}{s}\right)-\frac{1}{2}\left(3 G-\frac{\sigma_{u}}{s}\right) \cos \theta \\
P & =\frac{\psi}{\cos \theta}=\phi^{\prime}(s)-\frac{\left(3 G-\phi^{\prime}\right)(1-\cos \theta)}{2 \cos \theta}= \\
& =\frac{1}{2}\left(3 G+\phi^{\prime}\right)-\frac{3 G-\phi^{\prime}}{2 \cos \theta}
\end{aligned}
$$

with

$$
\begin{aligned}
& \cos \theta=\frac{\sigma_{i j} \delta \varepsilon_{i j}}{\sigma_{u} \delta s}, \quad \delta s=\frac{2}{\sqrt{3}}\left(\delta \varepsilon_{11}^{2}+\delta \varepsilon_{22}^{2}+\delta \varepsilon_{11} \delta \varepsilon_{22}+\delta \varepsilon_{12}^{2}\right)^{1 / 2} \\
& \delta \varepsilon_{i j}=-z \frac{\partial^{2} \delta W}{\partial x_{i} \partial x_{j}}, \quad(i, j, k, \ell=1,2) .
\end{aligned}
$$


In the same approach to the problem [2] the kinematic boundary conditions with edges simply supported are satisfied in the sense of Saint-Venant by chosen

$$
\delta W=C \cos \left(\frac{m \pi x_{1}}{a}+\frac{n \pi x_{2}}{b}\right)
$$

then

$$
\cos \theta=\frac{\sqrt{3}}{2} \frac{\left(p \frac{m^{2}}{a^{2}}+2 \tau \frac{m n}{a b}+q \frac{n^{2}}{b^{2}}\right)}{\left(p^{2}-p q+q^{2}+3 \tau^{2}\right)^{1 / 2}\left(\frac{m^{2}}{a^{2}}+\frac{n^{2}}{b^{2}}\right)}
$$

the quantities $A$ and $P$ are not depending on $z, x_{1}, x_{2}$, the stability equation reduces into the following

$$
\begin{gathered}
\alpha_{1} \frac{\partial^{4} \delta W}{\partial x_{1}^{4}}+\alpha_{2} \frac{\partial^{4} \delta W}{\partial x_{1}^{3} \partial x_{2}}+\alpha_{3} \frac{\partial^{4} \delta W}{\partial x_{1}^{2} \partial x_{2}^{2}}+\alpha_{4} \frac{\partial^{4} \delta W}{\partial x_{1} \partial x_{2}^{3}}+\alpha_{5} \frac{\partial^{4} \delta W}{\partial x_{2}^{4}}+ \\
+\frac{9}{A h^{2}}\left(p \frac{\partial^{2} \delta W}{\partial x_{1}^{2}}+2 \tau \frac{\partial^{2} \delta W}{\partial x_{1} \partial x_{2}}+q \frac{\partial^{2} \delta W}{\partial x_{2}^{2}}\right)=0
\end{gathered}
$$

where

$$
\begin{aligned}
& \alpha_{1}=1-\frac{3}{4}\left(1-\frac{P}{A}\right) \frac{p^{2}}{\sigma_{u}^{2}}, \quad \alpha_{2}=-3\left(1-\frac{P}{A}\right) \frac{p \tau}{\sigma_{u}^{2}}, \\
& \alpha_{3}=2-3\left(1-\frac{P}{A}\right) \frac{\tau^{2}}{\sigma_{u}^{2}}-\frac{3}{2}\left(1-\frac{P}{A}\right) \frac{p q}{\sigma_{u}^{2}}, \\
& \alpha_{4}=-3\left(1-\frac{P}{A}\right) \frac{q \tau}{\sigma_{u}^{2}}, \quad \alpha_{5}=1-\frac{3}{4}\left(1-\frac{P}{A}\right) \frac{q^{2}}{\sigma_{u}^{2}}
\end{aligned}
$$

Since there exists non - trivial solution $\delta W \neq 0$, i.e. $C \neq 0$, from the equations (1.4), (1.5) it follows a relation for defining critical loads

$$
i^{2}=9 \frac{b^{2}}{h^{2}}=\frac{\pi^{2} A\left(\alpha_{1} \frac{m^{4}}{a^{4}}+\alpha_{2} \frac{m^{3} n}{a^{3} b}+\alpha_{3} \frac{m^{2} n^{2}}{a^{2} b^{2}}+\alpha_{4} \frac{m n^{3}}{a b^{3}}+\alpha_{5} \frac{n^{4}}{b^{4}}\right)}{p \frac{m^{2}}{a^{2}}+2 \tau \frac{m n}{a b}+q \frac{n^{2}}{b^{2}}}
$$

For determining a combination of critical loads $p, q, \tau$ we apply the loading parameter method [1], suppose that the complex loading process is given, i.e. $p \equiv p(t), q \equiv q(t), r \equiv \tau(t)$ are known as functions of a loading parameter $t$. Therefore, a solution $s \equiv s(t)$ of the equation (1.1) with the initial condition $t=0, s=s_{0}$ can be found by Euler method or Runge - Kutta method. Using obtained result and solving the equation (1.6) gives a critical value of loading parameter $t_{*}$. Thus the critical loads are of the form

$$
q_{*}=q\left(t_{*}\right), \quad p_{*}=p\left(t_{*}\right), \quad \tau_{*}=\tau\left(t_{*}\right)
$$

\section{Remarks}

- If the elasto-plastic deformation process at the buckling moment is arbitrary complicated, the values $A, P$ are taken in the form $(1.3)$.

- If this process is less complicated, i.e. process with average curvature, replace $A, P$ by $\frac{\sigma_{u}}{s}$ and $\phi^{\prime}(s)$. 
- If this process is simple, replace $A, P$ by $E_{c}(s)$ and $E_{t}(s)$, where $E_{c}$ - secant modulus, $E_{t}$ - tangential modulus of stress versus strain characteristic, moreover $q=\beta_{1} p, \tau=\beta_{2} p, \sigma_{t}=\gamma p$, $\gamma=\left(1-\beta_{1}+\beta_{1}^{2}+3 \beta_{2}^{2}\right)^{1 / 2}$.

For illustration consider a square plate $(a=b)$ subjected to biaxial compressions and shear force, the minimal values of critical loads are obtained from (1.6) by putting $m=n=1$.

\section{§2. PLATE SUBJECTED TO BIAXIAL COMPRESSIONS}

In this case $\sigma_{11}=-p, \sigma_{22}=-q, \sigma_{12}=-\tau=0$. Analysis of the problem was given in $[1,3]$. For clearing up the influence of the complex loading process, we observe the case when it happens outside the yield stress surface. Critical loads are determined from the relation (1.6) according to

a) the elasto - plastic process theory

$$
\begin{aligned}
i^{2} \equiv & 9 \frac{a^{2}}{h^{2}}=\frac{\pi^{2}}{2(p+q)}\left[\left(3 G+\frac{\sigma_{u}}{s}\right)-\frac{\sqrt{3}}{4}\left(3 G-\frac{\sigma_{u}}{s}\right) \frac{p+q}{\sigma_{u}}\right] \times \\
& \times\left[4-\frac{3}{4}\left(1-\frac{\left(3 G+\phi^{\prime}\right)-\frac{4}{\sqrt{3}}\left(3 G-\phi^{\prime}\right) \frac{\sigma_{u}}{p+q}}{\left(3 G+\frac{\sigma_{u}}{s}\right)-\frac{\sqrt{3}}{4}\left(3 G-\frac{\sigma_{u}}{s}\right) \frac{p+q}{\sigma_{u}}}\right) \frac{(p+q)^{2}}{\sigma_{u}^{2}}\right]
\end{aligned}
$$

where $\sigma_{u}=\left(p-p q+q^{2}\right)^{1 / 2}$

b) the theory of process with average curvature [1]

$$
i^{2}=\frac{\pi^{2}\left(p^{2}-p q+q^{2}\right)^{1 / 2}}{s(p+q)}\left[4-\frac{3}{4}\left(1-\frac{s \phi^{\prime}(s)}{\left(p^{2}-p q+q^{2}\right)^{1 / 2}}\right) \frac{(p+q)^{2}}{p^{2}-p q+q^{2}}\right]
$$

c) the simple process theory

$$
i^{2}=\frac{\gamma_{1} \pi^{2}}{s\left(1+\beta_{1}\right)}\left[4-\frac{3}{4}\left(1-\frac{E_{t}(s)}{E_{c}(s)}\right) \frac{\left(1+\beta_{1}\right)^{2}}{\gamma_{\perp}^{2}}\right]
$$

with $\gamma_{1}=\left(1-\beta_{1}+\beta_{1}^{2}\right)^{1 / 2}$, then $p_{*}=\frac{E_{c}\left(s^{*}\right) s^{*}}{\gamma_{1}}, g_{*}=\beta_{1} p_{*}$.

A numerical comparison is established on the square plate made of steel 30XГCA and subjected to complex loading as follows (see fig. 1)

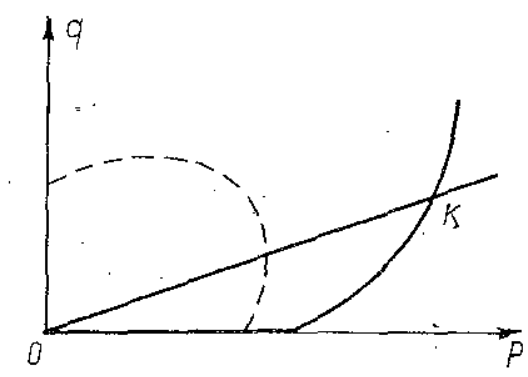

Fig. 1

$$
p(t)=p_{0} t+p_{1} t, \quad q(t)=q_{0} t^{2} ; \quad p_{0} \geq \sigma_{y}, t \geq 0
$$

with $p_{0}=4260 \mathrm{kG} / \mathrm{cm}^{2}, p_{1}=10 \mathrm{kG} / \mathrm{cm}^{2}, q_{0}=200 \mathrm{kG} / \mathrm{cm}^{2}, \sigma,=4000 \mathrm{kG} / \mathrm{cm}^{2}, 3 G=2.6$. $10^{6} \mathrm{kG} / \mathrm{cm}^{2} ; t=0, s_{0}=1.75 \cdot 10^{-3}$ 
The ratio $a / h$ varies from 22 to 43 . All calculations in solving problem on $\mathrm{PC}$ are fulfilled by Dao Thi Bich Hanh. Obtained results are presented on the tables: table 1 - critical loading parameter, critical loads calculated by the elasto plastic process theory (in solving equations (1.1) and (2.1)), table 2 - mentioned values by the theory of process with average curvature ((1.1) and (2.2)) and table 3 - by the simple process theory (2.3).

\section{Table 1}

\begin{tabular}{|c|c|c|c|c|c|}
\hline$a / h$ & s. $10^{3}$ & $t_{*}$ & $p_{*}$ & $q_{*}$ & $\sigma_{u}^{*}$ \\
\hline 22 & 3.52 & 2.871 & 4288 & 1648 & 3747 \\
\hline 25 & 3.136 & 2.563 & 4285 & 1313 & 3803 \\
\hline 28 & 2.8 & 2.291 & 4282 & 1049 & 3866 \\
\hline 31 & 2.53 & 2.038 & 4280 & 831 & 3931 \\
\hline 34. & 2.309 & 1.781 & 4277 & 634 & 3998 \\
\hline 37 & 2.108 & 1.479 & 4274 & 437 & 4073 \\
\hline 40 & 1.94 & 1.157 & 4271 & 267 & 4144 \\
\hline 43 & 1.82 & 0,754 & 4267 & 113 & 4211 \\
\hline
\end{tabular}

Tablè 2

\begin{tabular}{|c|c|c|c|c|c|}
\hline$a / h$ & $s .10^{3}$ & $t_{*}$ & $p_{*}$ & $q_{*}$ & $\sigma_{u}^{*}$ \\
\hline 22 & 3.54 & 2.890 & 4288 & 1670 & 3744 \\
\hline 25 & 3.16 & 2.584 & 4285 & 1335 & 3798 \\
\hline 28 & 2.838 & 2.320 & 4283 & 1076 & 3859 \\
\hline 31 & 2.57 & 2.082 & 4280 & 867 & 3920 \\
\hline 34 & 2.35 & 1.844 & 4278 & 680 & 3982 \\
\hline 37 & 2.17 & 1.584 & 4275 & 501 & 4048 \\
\hline 40 & 2.07 & 1.294 & 4272 & 335 & 4115 \\
\hline 43 & 1.88 & 0.985 & 4269 & 194 & 4176 \\
\hline
\end{tabular}

Table 9

\begin{tabular}{ccccccc}
$a / h$ & $s .10^{3}$ & & $p_{*}$ & $g_{*}$ & $\sigma_{u}^{*}$ \\
\hline & - & & & & \\
22 & 3.449 & & 5602 & 2152 & 4895 \\
25 & 3.116 & & 5428 & 1663 & 4817 \\
28 & 2.827 & & 5260 & 1288 & 4750 \\
31 & 2.583 & & 5081 & 986 & 4667 \\
34 & 2.382 & & 4906 & 727 & 4586 \\
37 & 2.222 & & 4741 & 484 & 4548 \\
40 & 2.062 & & 4607 & 288 & 4470 \\
43 & 1.936 & & 4459 & 118 & 4401 \\
\hline
\end{tabular}




\section{§3. PLATE SUBJECTED TO COMPRESSION AND SHEAR FORCE}

On this case $\sigma_{11}=-p, \sigma_{22}=0, \sigma_{12}=-\tau, \sigma_{u}=\left(p^{2}+3 t^{2}\right)^{1 / 2}$. Components of the strain velocity tensor are determined by the following equations

$$
\begin{aligned}
& \dot{\varepsilon}_{11}=-\frac{\dot{p}}{\sigma_{u} / s}-\left(\frac{1}{\phi^{\prime}(s)}-\frac{1}{\sigma_{u} / s}\right) \frac{p(p \dot{p}+3 \tau \dot{\tau})}{p^{2}+3 \tau^{2}}, \\
& \dot{\varepsilon}_{12}=-\frac{3 \dot{\tau}}{2 \sigma_{u} / s}-\frac{3}{2}\left(\frac{1}{\phi^{\prime}}-\frac{1}{\sigma_{u} / s}\right) \frac{\tau(p \dot{p}+3 \tau \dot{\tau})}{p^{2}+3 \tau^{2}}, \\
& \dot{\varepsilon}_{22}=-\dot{\varepsilon}_{11} / 2 .
\end{aligned}
$$

The arc - length of the strain trajectory is evaluated from

$$
\frac{d s}{d t}=\left(\dot{\varepsilon}_{11}^{2}+\frac{4}{3} \dot{\varepsilon}_{12}^{2}\right)^{1 / 2}=F(s, p, \tau)
$$

Finding the critical loads is suggested on the following relations respectively

a) the elasto - plastic process theory

$$
\begin{aligned}
i^{2}= & \frac{\pi^{2}}{2(p+2 \tau)}\left[\left(3 G+\frac{\sigma_{u}}{s}\right)-\frac{\sqrt{3}}{4}\left(3 G-\frac{\sigma_{u}}{s}\right) \frac{p+2 \tau}{\sigma_{u}}\right] \times \\
& \times\left[3-\frac{3}{4}\left(1-\frac{\left(3 G+\phi^{\prime}\right)-\frac{4}{\sqrt{3}}\left(3 G-\phi^{\prime}\right) \frac{\sigma_{u t}}{p+2 \tau}}{\left(3 G+\frac{\sigma_{u}}{s}\right)-\frac{\sqrt{3}}{4}\left(3 G-\frac{\sigma_{u}}{s}\right) \frac{p+2 \tau}{\sigma_{u}}} \frac{(p+2 \tau)^{2}}{\sigma_{u}^{2}}\right]\right.
\end{aligned}
$$

where $\sigma_{u}=\left(p^{2}+3 \tau^{2}\right)^{1 / 2}$.

b) the theory of process with average curvature

$$
i^{2}=\frac{\pi^{2}\left(p^{2}+3 r^{2}\right)^{1 / 2}}{(p+2 \tau) s}\left[3-\frac{3}{4}\left(1-\frac{s \phi^{\prime}(s)}{\left(p^{2}+3 \tau^{2}\right)^{1 / 2}}\right) \frac{(p+2 \tau)^{2}}{p^{2}+3 \tau^{2}}\right]
$$

c) the simple process theory

$$
i^{2}=\frac{\gamma_{2} \pi^{2}}{s\left(1+2 \beta_{2}\right)}\left[3-\frac{3}{4}\left(1-\frac{E_{t}(s)}{E_{c}(s)}\right) \frac{\left(1+2 \beta_{2}\right)^{2}}{\gamma_{2}^{2}}\right]
$$

with $\gamma_{2}=\left(1+3 \beta_{2}^{2}\right)^{1 / 2}$, then $p_{*}=\frac{E_{c}\left(s_{*}\right) s_{*}}{\gamma_{2}}, q_{*}=\beta_{2} p_{*}$.

A numerical analysis is considered on the square plate made of steel 30XГCA and subjected to complex loading process:

$$
\begin{aligned}
& p(t)=p_{0}+p_{1} t \\
& \tau(t)=\tau_{0} t^{2}, \quad p_{0} \geq \sigma_{y}, \quad t \geq 0
\end{aligned}
$$

with $p_{0}=4260 \mathrm{kG} / \mathrm{cm}^{2}, p_{1}=10 \mathrm{kG} / \mathrm{cm}^{2}, \tau_{0}=200 \mathrm{kG} / \mathrm{cm}^{2}$. The ratio $\mathrm{a} / \mathrm{h}$ varies from 22 to 37 . Dao Thi Bich Hanh has fulfilled all calculations in solving problem on PC. Obtained results are shown on the tables: table 4 - critical loading parameter, critical loads calculated by the elasto plastic process theory (in solving (3.2) and (3.3)); table 5 - by the theory of process with average curvature ((3.2) and (3.4)); table 6 - by the simple process theory (3.5). 


\begin{tabular}{lllllll}
\hline$a / h$ & $s .10^{3}$ & $t_{*}$ & $p_{*}$ & & $q_{*}$ & $\sigma_{u}^{*}$ \\
- & - & - & & & & - \\
22 & 2.6 & 1.955 & 4279 & 764 & 4479 \\
25 & 2.329 & 1.736 & 4277 & 602 & 4402 \\
28 & 2.11 & 1.462 & 4274 & 427 & 4338 \\
31 & 1.96 & 1.159 & 4271 & 268 & 4296 \\
34 & 1.85 & 0.828 & 4268 & 137 & 4274 \\
37 & 1.75 & 0.18 & 4261 & 6 & 4261 \\
\hline
\end{tabular}

Table 5

\begin{tabular}{llccccc}
\hline$a / h$ & $s .10^{3}$ & $t_{*}$ & & $p_{*}$ & $q_{*}$ & $\sigma_{u}^{*}$ \\
- & - & - & & - & - \\
22 & 3.095 & 2.19 & 4281 & 959 & 4592 \\
25 & 2.62 & 1.97 & 4279 & 776 & 4485 \\
28 & 2.33 & 1.737 & 4277 & 604 & 4403 \\
31 & 2.11 & 1.456 & 4274 & 423 & 4337 \\
34 & 1.95 & 1.144 & 4271 & 261 & 4295 \\
37 & 1.839 & 0.776 & 4276 & 120 & 4272 \\
\hline
\end{tabular}

Table 6

\begin{tabular}{llllll}
\hline$a / h$ & $s .10^{3}$ & $p_{*}$ & $q_{*}$ & $\sigma_{u}^{*}$ \\
- & - & - & & - \\
22 & 3.379 & & 4667 & 833 & 4885 \\
25 & 2.834 & & 4615 & 649 & 4756 \\
28 & 2.522 & 4568 & 456 & 4636 \\
31 & 2.297 & 4521 & 283 & 4547 \\
34 & 2.085 & 4472 & 143 & 4479 \\
37 & 1.944 & 4407 & 6 & 4407 \\
\hline
\end{tabular}

\section{§4. DISCUSSION}

From the above results we can lead some conclusions

a) Critical loads of the plate subjected to complex loading are always smaller than critical one when the simple loading happens. The workable possibility of structures with complex loading is less than that with simple loading.

b) The influence of the complex loading process on the critical loads is shown apparently when the process happens outside the yield stress surface. In the paper [3] the difference is not obvious, because the complex loading process happens inside the yield surface. 
c) In the stability problem of plate subjected to compression and shear force results obtained by theory of process with average curvature differ obviously from that one by elasto plastic process theory, since in this case the deformation process is more complicated.

d) In $[4,5]$ there are experimental data, the comparison can't be established, because the materials are different. But in the qualitative side this analysis gives an accordance with experimental data.

e) Elasto plastic deformation process plays an important role in the stability of structures, it requires to apply the theory of elasto plastic processes in solving the problem. This analysis may be highly reliable and may describe the real state of the structures.

This publication is completed with financial support from the National Basic Research Program in Natural Sciences.

\section{REFERENCES}

1. Dao Huy Bich. Influence of complex loading on the stability outside elastic limit of thin plates and shells. Journal of Mechanics, vol. 11, No 3, 1989 (in Vietnamese).

2. Dao Huy Bich. A new approach to the stability problem of plates subjected to arbitrary complex loading. Journal of Mechanics, vol. 14, No 2, 1992.

3. Dao Van Dung. On the stability of thin plates subjected to biaxial compressions. Journal of Mechanics, vol. 15, No 2, 1993 (in Vietnamese).

4. Zubtsaninov V. H. On the contemporary problems of inelastic stability. Selection: Stability in solid Mechanics. Kalinin 1981 (in Russian)

5. Garanikov V. V. Lotov V. N. Experimental research on buckling process of plates. Selection: Stability in solid Mechanics. Kalinin 1982 (in Russian).

Received September 28, 1999

\section{ỔN ĐINH DÂN DÉO CỦA BẢN DƯỚI TÁC DƯNG CỦA QUÁ TRÌNH ĐặT TẢI PHÚC TẠP}

Trong bài toán ổn định ngoài giới hạn đàn hò̀i quá trình đặt tải phức tạp lên vật thể có ảnh hưởng đáng kể đến giá trị tải tới hạn. Để làm sáng tó hiệu quá đó, trong bài này đã sư dụng lý thuyết quá trình đàn déo, lý thuyết quá trình độ cong trung bình và lý thuyết quá trình đặt tải đơn giản để xét bài toán nêu trên, khi độ phức tạp của quá trình xẩy ra ngoài mặt gịới hạn chảy. Đã trình bày so sánh kết quả băng số và nêu lên một số nhận xét. 